\title{
ON THE TOPOLOGICAL ENTROPY OF THE IRREGULAR PART OF V-STATISTICS MULTIFRACTAL SPECTRA
}

\author{
ALEJANDRO M. MESON AND FERNANDO VERICAT
}

INSTITUTO DE FISICA DE LIQUIDOS Y SISTEMAS BIOLOGICOS (IFLYSIB) CONICET LA PLATA - UNLP

AND

GRUPO DE APLICACIONES MATEMATICAS Y ESTADISTICAS DE LA FACULTAD DE INGENIERIA (GAMEFI), UNLP, LA PLATA, ARGENTINA

E-MAILS: MESON@IFLYSIB.UNLP.EDU.AR; VERICAT@IFLYSIB.UNLP.EDU.AR

(Received: 05 December 2012, Accepted: 05 January 2013)

Abstract. Let $(X, d)$ be a compact metric space and $f: X \rightarrow X$, if $X^{r}$ is the product of $r$-copies of $X, r \geq 1$, and $\Phi: X^{r} \rightarrow \mathbf{R}$, then the multifractal decomposition for $V$-statistics is given by

$$
E_{\Phi}(\alpha)=\left\{x: \lim _{n \rightarrow \infty} \frac{1}{n^{r}} \sum_{0 \leq i_{1} \leq \ldots \leq i_{r} \leq n-1} \Phi\left(f^{i_{1}}(x), \ldots, f^{i_{r}}(x)\right)=\alpha\right\} . \text { The }
$$

irregular part, or historic set, of the spectrum is the set points $x \in X$, for which the limit does not exist.

In this article we prove that for dynamical systems with specification, the irregular part of the $V$-statistics spectrum has topological entropy equal to that of the whole space $X$.

AMS Classification: 37C45, 37B40

Keywords: Topological entropy; $V$-statistics; Multifractal spectra

\section{INTRODUCTION}

Motivated by the problems on convergence of multiple ergodic averages Fan, Schmeling and $\mathrm{Wu}[5]$, treated the problem of multifractal analysis of $V$-statistics.

JOURNAL OF DYNAMICAL SYSTEMS \& GEOMETRIC THEORIES VOL. 11, NUMBER 1(2013) 1-12.

(C) TARU PUBLICATIONS 
In the present paper, we would like to study the irregular part of the multifractal decomposition.

Let us consider a topological dynamical system $(X, f)$, with $X$ a compact metric space and $f$ a continuous map. Let $X^{r}=X \times \ldots \times X$ be the product of $r$-copies of $X$ with $r \geq 1$, if $\Phi: X^{r} \rightarrow \mathbf{R}$ is a continuous map, then let

$$
V_{\Phi}(n, x)=\frac{1}{n^{r}} \sum_{1 \leq i_{1}, \ldots, i_{r} \leq n} \Phi\left(f^{i_{1}}(x), \ldots, f^{i_{r}}(x)\right) .
$$

These averages are called the $V$-statistics of order $r$ with kernel $\Phi$. For the idea of $V$-statistics from a Statistical point of view and its relationship with the $U$-statistics see section 2 of $[5]$

Ergodic limits of the form

$$
\lim _{n \rightarrow \infty} \frac{1}{n} \sum_{i=0}^{n-1} \Phi\left(f^{i_{1}}(x), \ldots, f^{i_{r}}(x)\right),
$$

were studied among others by Furstenberg[8], Bergelson[2] and Bourgain[3].

The multifractal spectra of $V$-statistics are specified by the decomposition sets

$$
E_{\Phi}(\alpha)=\left\{x: \lim _{n \rightarrow \infty} V_{\Phi}(n, x)=\alpha\right\} .
$$

Fan, Schemeling and $\mathrm{Wu}[5]$ treated the problem of measuring the sizes of the multifractal sets $E_{\Phi}(\alpha)$. They established the following variational principle:

$$
h_{\text {top }}\left(E_{\Phi}(\alpha)\right)=\sup \left\{h_{\mu}(f): \int \Phi d \mu^{\otimes r}=\alpha\right\},
$$

where $h_{\mu}$ is the measure-theoretic entropy of $\mu$. This formula is valid for dynamical systems with the specification property. This generalizes the variational formula obtained by Takens and Verbitski for $r=1[9]$.

The irregular part of the spectrum, or historic set, is the set of points $x$ for which $\lim _{n \rightarrow \infty} V_{\Phi}(n, x)$ does not exist. We denote this set by $E_{\Phi}^{\infty}$, so that the space $X$ can be decomposed as

$$
X=\bigcup_{\alpha \in \mathbf{R}} E_{\Phi}(\alpha) \cup E_{\Phi}^{\infty}
$$


ON THE TOPOLOGICAL ENTROPY OF THE .. — JDSGT VOL. 11, NUMBER 1 (2013) 3

An important problem in Multifractal Analysis is to determine the dimension of the irregular part. For $r=1$ the irregular part of the spectrum has been extensively studied. Fan, Feng and Wu, in reference [6], did it for topological mixing subshifts. Barreira and Schmeling[1] obtained a similar result than [6] but for Hölder continuous maps. More recently the irregular part was studied by Thompson [11] and by Zhou and Chen [13]. Here we propose the study of the irregular part of the spectrum for multiple ergodic averages. The result to be proved is

Theorem: Let $(X, f)$ be a dynamical system with the property of specification, let $\Phi \in C\left(X^{r}\right), r \geq 1$, if the irregular part $E_{\Phi}^{\infty}$ of the spectrum of multiple ergodic averages $V_{\Phi}(n, x)$ is non-empty then it has the same topological entropy as the whole space $X$.

The case $E_{\Phi}^{\infty}=\varnothing$ can occur in situations like for instance $\Phi$ cohomologous to 0 , or when the ergodic limits $V_{\Phi}(n, x)$ have the same value for any $x$.

\section{Preliminary Definitions}

Firstly let us recall the Bowen definition of topological entropy of sets: Let $f: X \rightarrow X$, with $X$ a compact metric space, for $n \geq 1$ the dynamical metric, or Bowen metric, is $d_{n}(x, y)=\max \left\{d\left(f^{i}(x), f^{i}(y)\right): i=0,1, \ldots, n-1\right\}$. We denote by $B_{n, \varepsilon}(x)$ the ball of centre $x$ and radius $\varepsilon$ in the metric $d_{n}$. Let $Z \subset X$ and let $\mathcal{C}(n, \varepsilon, Z)$ be the collection of finite or countable coverings of the set $Z$ by balls $B_{m, \varepsilon}(x)$ with $m \geq n$. Let

$$
M(Z, s, n, \varepsilon)=\inf _{\mathcal{B} \in \mathcal{C}(n, \varepsilon, Z)} \sum_{B_{m, \varepsilon}(x) \in \mathcal{B}} \exp (-s m),
$$

and set

$$
M(Z, s, \varepsilon)=\lim _{n \rightarrow \infty} M(Z, s, n, \varepsilon) .
$$

There is an unique number $\bar{s}$ such that $M(Z, s, \varepsilon)$ jumps from $+\infty$ to 0 . Let

$$
H(Z, \varepsilon)=\bar{s}=\sup \{s: M(Z, s, \varepsilon)=+\infty\}=\inf \{s: M(Z, s, \varepsilon)=0\},
$$

and

$$
h_{t o p}(Z)=\lim _{\varepsilon \rightarrow 0} H(Z, \varepsilon)
$$


The number $h_{\text {top }}(Z)$ is the topological entropy of $Z$.

A dynamical system $(X, f)$ has the specification property if the following condition holds: for $\varepsilon>0$, there is an integer $M(\varepsilon)$ such that for any finite disjoint collection of integer intervals $I_{1}=\left[a_{1}, b_{1}\right], \ldots, I_{k}=\left[a_{k}, b_{k}\right]$, of length $\geq M(\varepsilon)$ and for any points $x_{1}, x_{2}, \ldots, x_{k} \in X$, there is a point $z \in X$ which $\varepsilon$-shadows the sequence $\left\{x_{1}, x_{2}, \ldots, x_{k}\right\}$, i.e. $d\left(f^{a_{j}+n}(z), f^{n}\left(x_{j}\right)\right) \leq \varepsilon$, for any $n=0, . ., b_{j}-a_{j}$ and $j=0,1, \ldots, k$.

By $\mathcal{M}(X)$ we denote the space of measures in $X$, and by $\mathcal{M}_{\text {inv }}(X, f)$ the space of $f$-invariant measures on $X$. The space $\mathcal{M}(X)$ can be endowed with a metric $D$ compatible with the metric in $X$, in the sense that $D\left(\delta_{x}, \delta_{y}\right)=d(x, y)$, where $\delta$ is the point mass measure. More precisely the metric considered in $\mathcal{M}(X)$ will be

$$
D(\mu, \nu)={ }_{n=1}^{\infty} \frac{\left|\int \varphi_{n} d \mu-\int \varphi_{n} d \nu\right|}{2^{n}\left\|\varphi_{n}\right\|_{\infty}},
$$

where $\left\{\varphi_{n}\right\}$ is a dense set in $C(X)$. We denote by $B_{R}(\mu)$ the ball of center $\mu$ and radius $R$ in the above metric. The topology induced by this metric is the weak star topology, and if $X$ is compact then $\mathcal{M}(X)$ is compact in the weak topology. The weak star convergence is the convergence in the metric which induces the weak star topology.

The so called empirical measures on $X$ associated to the dynamical system $(X, f)$ are

$$
\mathcal{E}_{n}(x)=\frac{1}{n} \sum_{i=0}^{n-1} \delta_{f^{i}(x)} .
$$

We denote the weak limits of the sequence $\left\{\mathcal{E}_{n}(x)\right\}$ by $V(x)$. Since $X$ is compact, $V(x) \neq \varnothing$. If $\mu$ is a measure on $X$ then a point $x \in X$ is $\mu$-generic if $V(x)=\{\mu\}$, by $G(\mu)$ is denoted the set of $\mu$-generic points. A result by Bowen[4] is that if $\mu$ is ergodic then

$$
h_{t o p}(G(\mu))=h_{\mu}(f)
$$

For general measures, not necessarily ergodic, the equality holds for dynamical systems with the specification property[7]. This result is the key point in the proof of variational theorem of Fan, Schemeling and $\mathrm{Wu}[5]$. 
ON THE TOPOLOGICAL ENTROPY OF THE _.. — JDSGT VOL. 11, NUMBER 1 (2013) 5

\section{Proof of THE THEOREM}

Let

$$
\mathcal{M}_{\Phi}(\alpha)=\left\{\mu \in \mathcal{M}_{i n v}(X): \int \Phi d \mu^{\otimes r}=\alpha\right\}
$$

and let

$G_{\Phi}(\alpha)=\left\{x:\right.$ there is $\left\{n_{k}\right\}$ such that $\left.w^{*}-\lim _{k \rightarrow \infty} \mathcal{E}_{n_{k}}(x)=\mu \in \mathcal{M}_{\Phi}(\alpha)\right\}$,

here $w^{*}-$ means weak star convergence.

For $\alpha_{1} \neq \alpha_{2} \in \mathbf{R}$, we shall find a set $G \subset G_{\Phi}\left(\alpha_{1}\right) \cap G_{\Phi}\left(\alpha_{2}\right)$.

Before proving the theorem we give some lemmas.

Lemma 1: If $\alpha_{1} \neq \alpha_{2}$ then $G_{\Phi}\left(\alpha_{1}\right) \cap G_{\Phi}\left(\alpha_{2}\right) \subset E_{\Phi}^{\infty}$.

Proof: In [5] was established, as a consequence of the Stone-Weierstrass theorem, that for any $\Phi \in C\left(X^{r}\right)$ and for any $\varepsilon>0$ there is a map $\widetilde{\Phi}: X^{r} \rightarrow \mathbf{R}$ of the form

$$
\widetilde{\Phi}=\sum_{j} \varphi_{j}^{(1)} \otimes \ldots \otimes \varphi_{j}^{(r)}
$$

with $\varphi_{j}^{(i)} \in C(X)$ such that $\|\Phi-\widetilde{\Phi}\|_{\infty}<\varepsilon$. Let $x \in G_{\Phi}\left(\alpha_{1}\right) \cap G_{\Phi}\left(\alpha_{2}\right)$, so there are sequences $\left\{n_{k}\right\},\left\{m_{k}\right\}$ such that

$$
\begin{aligned}
& \mu=w^{*}-\lim _{k \rightarrow \infty} \mathcal{E}_{n_{k}}(x) ; \mu \in \mathcal{M}_{\Phi}\left(\alpha_{1}\right) \\
& \nu=w^{*}-\lim _{k \rightarrow \infty} \mathcal{E}_{m_{k}}(x) ; \nu \in \mathcal{M}_{\Phi}\left(\alpha_{2}\right),
\end{aligned}
$$

We have

$$
V_{\widetilde{\Phi}}(n, x)=\sum_{j} \prod_{i=1}^{r} \frac{1}{n} S_{n}\left(\varphi_{j}^{(i)}(x)\right)
$$

where $S_{n}\left(\varphi_{j}^{(i)}(x)\right)=\sum_{k=0}^{n-1} \varphi_{j}^{(i)}\left(f^{k}(x)\right)$. Therefore, by Eqs.(3-4) 


$$
\begin{aligned}
\lim _{k \rightarrow \infty} V_{\widetilde{\Phi}}\left(n_{k}, x\right) & =\int \widetilde{\Phi} d \mu^{\otimes r} \\
\lim _{k \rightarrow \infty} V_{\widetilde{\Phi}}\left(m_{k}, x\right) & =\int \widetilde{\Phi} d \nu^{\otimes r} .
\end{aligned}
$$

By the above argument of approximation we get in the same way of [5] that

$\lim _{k \rightarrow \infty} V_{\Phi}\left(n_{k}, x\right)=\int \Phi d \mu^{\otimes r}=\alpha_{1}$ and $\lim _{k \rightarrow \infty} V_{\Phi}\left(m_{k}, x\right) \int \Phi d \nu^{\otimes r}=\alpha_{2}$, with $\alpha_{1} \neq \alpha_{2}$.

Then $x \in E_{\Phi}^{\infty}$.

We have that

$$
G_{\Phi}(\alpha) \subset\left\{x: \exists \mu \in V(x), \text { such that } h_{\mu}(f) \leq \sup \left\{h_{\mu}(f): \int \Phi d \mu^{\otimes r}=\alpha\right\}\right\}
$$

and so, by the Bowen lemma

$$
h_{\text {top }}\left(G_{\Phi}(\alpha)\right) \leq \sup \left\{h_{\mu}(f): \int \Phi d \mu^{\otimes r}=\alpha\right\}
$$

For $\rho_{1}, \rho_{2}, \ldots, \rho_{k} \in \mathcal{M}(X)$ and positive numbers $R_{1}, R_{2}, \ldots, R_{k}$, let $x_{1}, x_{2}, \ldots, x_{k} \in$ $X, n_{1}, n_{2}, \ldots, n_{k} \in \mathbf{N}$ such that $\mathcal{E}_{n_{j}}\left(x_{j}\right) \in B_{R_{j}}\left(\rho_{j}\right), j=1,2, \ldots, k$, for a given $\rho_{1}, \rho_{2}, \ldots, \rho_{k} \in \mathcal{M}(X)$ and $R_{1}, R_{2}, \ldots, R_{k}$. Let $\varepsilon_{1}>0, \varepsilon_{2}>0, \ldots, \varepsilon_{k}>0$, if $n_{i}>$ $M\left(\varepsilon_{i}\right)$ (the number of specification), $i=1,2, \ldots, k$, then by specification property

$$
\bigcap_{j=1}^{k} f^{-M_{j-1}}\left(B_{n_{j}, \varepsilon_{j}}\left(x_{j}\right)\right) \neq \varnothing, \text { with } M_{j}=n_{1}+n_{2}+\ldots+n_{j} .
$$

Lemma 2: Let $z \in \bigcap_{j=1}^{k} f^{-M_{j-1}}\left(B_{n_{j}, \varepsilon_{j}}\left(x_{j}\right)\right)$, then for any $\rho \in \mathcal{M}(X)$ holds

$$
D\left(\mathcal{E}_{M_{k}}(z), \rho\right) \leq \frac{1}{M_{k}} \sum_{j=1}^{k} n_{j}\left(\overline{R_{j}}+D\left(\rho_{j}, \rho\right)\right)
$$

where $\overline{R_{j}}=R_{j}+\varepsilon_{j}, j=1,2, \ldots, k .$. ,

Remark: It can replace an uniform $\varepsilon$ for all balls $B_{n_{j}, \varepsilon}\left(x_{j}\right)$, by the $\varepsilon_{1}, \varepsilon_{2}, \ldots, \varepsilon_{k}$, following a trick used in the proof of the proposition 2.1 in [10].

Proof: We have 
ON THE TOPOLOGICAL ENTROPY OF THE ... — JDSGT VOL. 11, NUMBER 1 (2013) 7

$$
\mathcal{E}_{M_{k}}(z)=\frac{1}{M_{k}} \sum_{j=1}^{k} n_{j} \mathcal{E}_{n_{j}}\left(f^{M_{j-1}}(z)\right)
$$

and

$$
D\left(\mathcal{E}_{n_{j}}\left(x_{j}\right), \mathcal{E}_{n_{j}}\left(f^{M_{j-1}}(z)\right)\right) \leq \frac{1}{n_{j}} \sum_{l=0}^{n_{j}-1} d\left(f^{l}\left(x_{j}\right), f^{-M_{j-1}-l}(z)\right) .
$$

Therefore

$$
\begin{aligned}
& D\left(\mathcal{E}_{M_{k}}(z), \rho\right) \\
\leq & \frac{1}{M_{k}} \sum_{j=1}^{k}\left[D\left(\mathcal{E}_{n_{j}}\left(x_{j}\right),{ }_{j}, \mathcal{E}_{n_{j}}\left(f^{M_{j-1}}(z)\right)\right)+D\left(\mathcal{E}_{n_{j}}\left(x_{j}\right), \rho_{j}\right)+D\left(\rho_{j}, \rho\right)\right] \\
\leq & \frac{1}{M_{k}} \sum_{j=1}^{k}\left[R_{j}+\varepsilon_{j}+D\left(\rho_{j}, \rho\right)\right]
\end{aligned}
$$

Lemma 3: Let $\alpha_{1} \neq \alpha_{2}$ with $\mathcal{M}_{\Phi}\left(\alpha_{1}\right) \neq \varnothing, \mathcal{M}_{\Phi}\left(\alpha_{2}\right) \neq \varnothing$ then

$$
h_{\text {top }}\left(G_{\Phi}\left(\alpha_{1}\right) \cap G_{\Phi}\left(\alpha_{2}\right)\right)=\min \left\{h_{\text {top }}\left(G_{\Phi}\left(\alpha_{1}\right)\right), h_{\text {top }}\left(G_{\Phi}\left(\alpha_{2}\right)\right)\right\} \text {. }
$$

Proof: Since $G_{\Phi}\left(\alpha_{1}\right) \cap G_{\Phi}\left(\alpha_{2}\right) \subset G_{\Phi}\left(\alpha_{1}\right)$ and $G_{\Phi}\left(\alpha_{1}\right) \cap G_{\Phi}\left(\alpha_{2}\right) \subset G_{\Phi}\left(\alpha_{2}\right)$, by the monotonicity of the entropy we have

$$
h_{\text {top }}\left(G_{\Phi}\left(\alpha_{1}\right) \cap G_{\Phi}\left(\alpha_{2}\right)\right) \leq \min \left\{h_{\text {top }}\left(G_{\Phi}\left(\alpha_{1}\right)\right), h_{\text {top }}\left(G_{\Phi}\left(\alpha_{2}\right)\right)\right\}
$$

. To prove the other inequality we shall find a set $G \subset G_{\Phi}\left(\alpha_{1}\right) \cap G_{\Phi}\left(\alpha_{2}\right)$ with $h_{\text {top }}(G) \geq \min \left\{h_{\text {top }}\left(G_{\Phi}\left(\alpha_{1}\right)\right), h_{\text {top }}\left(G_{\Phi}\left(\alpha_{2}\right)\right)\right\}$.

To construct $G$, let us choose sequences $\left\{n_{k}\right\},\left\{R_{k}\right\},\left\{\varepsilon_{k}\right\}$ with $R_{k} \searrow 0$ and $\varepsilon_{k} \searrow 0$ and, for a given sequence $\left\{\rho_{1}, \rho_{2}, \ldots, \rho_{k}\right\} \subset \mathcal{M}(X)$, for, $\bar{\varepsilon}>\varepsilon_{1}$.let us consider $\left(n_{k}, \bar{\varepsilon}\right)$-sets $\Gamma_{k} \subset\left\{x: \mathcal{E}_{n_{k}}(x) \in B_{R_{k}}\left(\rho_{k}\right)\right\}$, so that (by the Lemma 2)

$$
x \in \Gamma_{k}, z \in B_{n_{k}, \varepsilon_{k}}(x) \Longrightarrow \mathcal{E}_{n_{k}}(z) \in B_{R_{k}+\varepsilon_{k}}\left(\rho_{k}\right) .
$$

Let us choose now a strictly increasing sequence $\left\{N_{k}\right\}$ such that 


$$
n_{k+1} \leq R_{k} \sum_{j=1}^{k} n_{j} N_{j}
$$

and

$$
\sum_{j=1}^{k-1} n_{j} N_{j} \leq R_{k} \sum_{j=1}^{k} n_{j} N_{j}
$$

We consider stretched sequences $\left\{n_{j}^{\prime}\right\},\left\{\varepsilon_{j}^{\prime}\right\},\left\{\Gamma_{j}^{\prime}\right\}$ such that if $j=N_{1}+\ldots+$ $N_{k-1}+q$ with $1 \leq q \leq N_{k}$ then $n_{j}^{\prime}=n_{k}, \quad \varepsilon_{j}^{\prime}=\varepsilon_{k}$ and $\Gamma_{j}^{\prime}=\Gamma_{k}$.

Finally, we can define

$$
G_{k}:=\bigcap_{j=1}^{k}\left(\bigcup_{x_{j} \in \Gamma_{j}^{\prime}} f^{-M_{j-1}}\left(B_{n_{j}^{\prime}, \varepsilon_{j}^{\prime}}\left(x_{j}\right)\right)\right),
$$

with $M_{j}=n_{1}^{\prime}+n_{2}^{\prime}+\ldots+n_{j}^{\prime}$ and

$$
G:=\bigcap_{k \geq 1} G_{k} .
$$

Any element of $G$ can be labelled by a sequence $x_{1} x_{2} \ldots$, with $x_{j} \in \Gamma_{j}^{\prime}$. According to Pfister and Sullivan [10] the following holds: Let $x_{j}, y_{j} \in \Gamma_{j}^{\prime}, x_{j} \neq y_{j}$, if $x \in$ $B_{n_{j}, \epsilon_{j}}\left(x_{j}\right), y \in B_{n_{j}, \epsilon_{j}}\left(y_{j}\right)$ then $\max \left\{d\left(f^{k}(x), f^{k}(y)\right): k=0, \ldots, n_{j}-1\right\}>2 \varepsilon$, with $\varepsilon>\varepsilon_{1} / 4$.

We see that $G \subset G_{\Phi}\left(\alpha_{1}\right) \cap G_{\Phi}\left(\alpha_{2}\right)$. Let $z \in G$, and let $\mu_{0} \in \mathcal{M}_{\Phi}\left(\alpha_{1}\right), \nu_{0} \in$ $\mathcal{M}_{\Phi}\left(\alpha_{2}\right)$, it can be considered sequences[13] $\left\{\mu_{k}\right\},\left\{\nu_{k}\right\}$ such that $D\left(\mu_{0}, \mu_{k}\right)<R_{k}$ and $D\left(\nu_{0}, \nu_{k}\right)<R_{k}$, then form the sequence

$$
\left\{\rho_{k}\right\}=\left\{\mu_{1}, \mu_{1}, \nu_{1}, \nu_{1}, \mu_{2}, \mu_{2}, \nu_{2}, \nu_{2}, \ldots\right\} .
$$

Let $\rho \in\left\{\mu_{0}, \nu_{0}\right\}$, and $\sum_{l=1}^{j} n_{l} N_{l} \leq M_{k} \leq \sum_{l=1}^{j+1} n_{l} N_{l}$, thus

Therefore

$$
\begin{gathered}
D\left(\mathcal{E}_{M_{k}}(z), \rho\right) \leq \frac{1}{M_{k}} \sum_{l=1}^{j-1} n_{l} N_{l} D\left(\mathcal{E}_{\sum_{l=1} n_{l} N_{l}}(z), \rho\right)+\frac{n_{j} N_{j}}{M_{k}} D\left(\mathcal{E}_{n_{j} N_{j}}(z), \rho\right)+ \\
\frac{M_{k}-\sum_{l=1}^{j} n_{l} N_{l j}}{M_{k}} D\left(\mathcal{E}_{n_{j+1} N_{j+1}}(z), \rho\right) .
\end{gathered}
$$


ON THE TOPOLOGICAL ENTROPY OF THE _.. — JDSGT VOL. 11, NUMBER 1 (2013) 9

$$
\begin{aligned}
& D\left(\mathcal{E}_{M_{k}}(z), \rho\right) \\
\leq & R_{j}+D\left(\mathcal{E}_{n_{j} N_{j}}(z), \rho_{j}\right)+D\left(\rho_{j}, \rho\right)+D\left(\mathcal{E}_{n_{j+1} N_{j+1}}(z), \rho\right)+D\left(\rho_{j+1}, \rho\right) \\
\leq & 2 R_{j}+\varepsilon_{j}+D\left(\rho_{j}, \rho\right)+D\left(\rho_{j+1}, \rho\right) .
\end{aligned}
$$

Thus, choosing subsequences $t_{k}=4 k+1$ and $s_{k}=4 k+3$, we get

$$
\begin{aligned}
& \mu_{0}=w^{*}-\lim _{k \rightarrow \infty} \mathcal{E}_{M_{t_{k}}}(z) \\
& \nu_{0}=w^{*}-\lim _{k \rightarrow \infty} \mathcal{E}_{M_{s_{k}}}(z),
\end{aligned}
$$

so that $z \in G_{\Phi}\left(\alpha_{1}\right) \cap G_{\Phi}\left(\alpha_{2}\right)$.

To complete the proof it must be proved that

$$
h_{\text {top }}(G) \geq \min \left\{h_{\text {top }}\left(G_{\Phi}\left(\alpha_{1}\right)\right), h_{\text {top }}\left(G_{\Phi}\left(\alpha_{2}\right)\right)\right\} .
$$

For this, we follow [10]. Let $s<\bar{h}:=\min \left\{h_{\text {top }}\left(G_{\Phi}\left(\alpha_{1}\right)\right), h_{\text {top }}\left(G_{\Phi}\left(\alpha_{2}\right)\right)\right\}$, the set $G$ is closed, and so it is compact, let us consider a finite covering $\mathcal{U}$ by balls $B_{m, \varepsilon}(x)$ having non-empty intersection with $G$. Now

$$
M(G, s, N, \varepsilon)=\inf _{\mathcal{U} \in \mathcal{C}(n, \varepsilon, G)} \sum_{B_{m, \varepsilon}(x) \in \mathcal{U}} \exp (-s m)
$$

For any finite covering $\mathcal{U}$ of $G$, we can construct a covering $\mathcal{U}_{0}$ in the following way: each ball $B_{m, \varepsilon}(x)$ is replaced by a ball $B_{M_{r r}, \varepsilon}(x)$ with $M_{r} \leq m \leq M_{r+1}$. Thus

$$
M(G, s, N, \varepsilon)=\inf _{\mathcal{U} \in \mathcal{C}(n, \varepsilon, G)} \sum_{B_{m, \varepsilon}(x) \in \mathcal{U}} \exp (-s m) \geq \inf _{\mathcal{U} \in \mathcal{C}(N, \varepsilon, G)} \sum_{B_{M_{r}, \varepsilon} \in \mathcal{U}_{0}} \exp \left(-s M_{r+1}\right) .
$$

Now we can consider a covering $\mathcal{U}_{0}$ in which

$$
m=\max \left\{r: \text { there is a ball } B_{M_{r}, \varepsilon}(x) \in \mathcal{U}_{0}\right\} .
$$

We set

$$
W_{k}:=\prod_{i=1}^{k} \Gamma_{i}, \quad \overline{W_{m}}=\bigcup_{k=1}^{m} W_{k}
$$


Let $x_{j}, y_{j} \in \Gamma_{j}^{\prime}, x_{j} \neq y_{j}$, as we pointed out earlier, if $x \in B_{N_{j}^{\prime}, \varepsilon_{j}^{\prime}}\left(x_{j}\right), y \in$ $B_{N_{j}^{\prime}, \varepsilon_{j}^{\prime}}\left(y_{j}\right)$ then $d\left(f^{l}(x), f^{l}(y)\right)>2 \varepsilon$

for any $l=0, \ldots, N_{j}-1$, and with $\varepsilon>\varepsilon_{1} / 4$. Now for any $x \in B_{M_{r}, \varepsilon}(z) \cap G$ there is a, uniquely determined $z=z(x) \in W_{r}$. A word $\bar{w} \in W_{j}$, with $j=1,2, \ldots, k$, is a called a prefix of a word $w \in W_{k}$ if the first $j$-letters of $\bar{w}$ agree with the first $j$-letters of $w$. The number of times that each $w \in W_{k}$ is a prefix of a word in $W_{m}$ is

$\operatorname{card} W_{m} / \operatorname{card} W_{k}$, thus if $W$ is a subset of $\overline{W_{m}}$ then

$$
\sum_{k=1}^{m} \frac{\operatorname{card}\left(W \cap W_{k}\right)}{\operatorname{card}\left(W_{k}\right)} \geq \operatorname{card}\left(W_{m}\right) .
$$

If each word in $W_{m}$ has a prefix contained in a $W \subset \overline{W_{m}}$ then

$$
\sum_{k=1}^{m} \frac{\operatorname{card}\left(W \cap W_{k}\right)}{\operatorname{card}\left(W_{k}\right)} \geq 1,
$$

and since $\mathcal{U}_{0}$ is a covering each point of $W_{m}$ has a prefix associated to a ball in $\mathcal{U}_{0}$. By this and because $c a r d W_{k} \geq \exp \left(\bar{h} M_{r}\right)$, we obtain

$$
\sum_{B_{M_{r}, \varepsilon} \in \mathcal{U}_{0}} \exp \left(-s M_{r}\right) \geq 1 .
$$

Thus if $r$ is taken such that $k \geq r$ then $s M_{k+1} \leq \bar{h} M_{k}$, for $N \geq M_{r}, \mathcal{U} \in \mathcal{G}(N, \varepsilon, G)$.

Therefore

$$
\sum_{B_{m, \varepsilon}(x) \in \mathcal{U}} \exp (-s m) \geq 1
$$

and so

$$
M(G, s, N, \varepsilon) \geq 1 .
$$

By this $h_{t o p}(G) \geq \bar{h}$.

We are now in condition of giving the proof of the theorem. Let 
ON THE TOPOLOGICAL ENTROPY OF THE _.. — JDSGT VOL. 11, NUMBER 1 (2013 ) 11

$$
\begin{aligned}
\Psi & =\Psi_{r, \Phi}: \mathcal{M}(X) \rightarrow \mathbf{R} \\
\Psi(\mu) & =\int \Phi d \mu^{\otimes r}
\end{aligned}
$$

and let

$h=h_{\text {top }}(X)$ be the topological entropy of the whole space $X$. By the classical variational principle and by the variational principle of [5]

$$
\begin{aligned}
& h=\sup \left\{h_{\mu}(f): \mu \in \mathcal{M}_{i n v}(X, f)\right\}=\sup _{\alpha \in \operatorname{Im}(\Psi)}\left\{h_{\mu}(f): \mu \in \mathcal{M}_{\Phi}(\alpha)\right\}= \\
& \sup _{\alpha \in \operatorname{Im}(\Psi)}\left\{h_{\text {top }}\left(E_{\Phi}(\alpha)\right)\right\} .
\end{aligned}
$$

We must show that $h_{\text {top }}\left(E_{\Phi}^{\infty}\right) \geq h$. For any $\gamma>0$, there is an $\alpha_{1} \in \operatorname{Im} \Psi$ such that $h_{\text {top }}\left(E_{\Phi}\left(\alpha_{1}\right)\right)>h-\gamma$, let $\alpha_{2} \in \operatorname{Im} \Psi$ and let $\mu_{1}, \mu_{2} \in \mathcal{M}(X, f)$ with $\Psi\left(\mu_{1}\right)=\alpha_{1}$, $\Psi\left(\mu_{2}\right)=\alpha_{2}$. The map $\lambda \longmapsto \Psi\left((1-\lambda) \mu_{1}+\lambda \mu_{2}\right)$ is continuous.Recall that

$$
\begin{aligned}
& h_{\text {top }}\left(G_{\Phi}\left(\alpha_{1}\right) \cap G_{\Phi}\left((1-\lambda) \alpha_{1}+\lambda \alpha_{2}\right)\right) \\
= & \min \left\{h_{\text {top }}\left(G_{\Phi}\left(\alpha_{1}\right), h_{t o p}\left(G_{\Phi}\left((1-\lambda) \alpha_{1}+\lambda \alpha_{2}\right)\right)\right\},\right.
\end{aligned}
$$

then, by the continuity of $\Psi$ as a function of $\lambda$, we have

$$
\begin{aligned}
h_{\text {top }}\left(E_{\Phi}^{\infty}\right) & \geq \lim _{\lambda \rightarrow 0} h_{\text {top }}\left(G_{\Phi}\left(\alpha_{1}\right) \cap G_{\Phi}\left((1-\lambda) \alpha_{1}+\lambda \alpha_{2}\right)\right) \geq \\
h_{\text {top }}\left(G_{\Phi}\left(\alpha_{1}\right)\right. & \geq h_{\text {top }}\left(E_{\Phi}\left(\alpha_{1}\right)\right)>h-\gamma .
\end{aligned}
$$

Since $\gamma$ is arbitrary the result follows.

We wish to acknowledge the referees for the valuable remarks and suggestions to improve this paper. Support of this work by Consejo Nacional de Investigaciones Científicas y Técnicas (PIP 112-200801-01192 ), Universidad Nacional de La Plata (Grant 11/I108) and Agencia Nacional de Promoción Científica y Tecnológica of Argentina (PICT 2007-00908) is greatly appreciated. F.V. is a member of CONICET. 


\section{REFERENCES}

[1] L- Barreira and J. Schmeling, Invariant sets with zero measure and full Hausdorff dimension, Electron. Res. Announc. Amer. Math. Soc. 3 (1997), 114-118

[2] V. Bergelson, Weakly mixing PET, Ergod. Th. and Dynam. Sys. 7, (1987) 337-349.

[3] J. Bourgain, Double recurrence and almost sure convergence, J. Reine Angew Math 404, (1990) 140-161

[4] R. Bowen, Topological entropy for non-compact sets, Trans. Amer. Math. Soc., 184, (1973) 125-136.

[5] A. H. Fan, J. Schmeling and J. Wu, The multifractal spectrta of $V$-statistics, preprint, arXiv:1206.3214v1 (2012)

[6] A. Fan, D. J. Feng and J. Wu , Recurrence, dimension and entropy, J. London. Math. Soc., 64, (2001) 229-244.

[7] A. Fan, I. M. Liao and J.Peyrière, Generic points in systems of specification and Banach valued Birkhoff averages, Disc. Cont. Dynam. Sys. 21, (2008) 1103-1128.

[8] H. Furstenberg, Ergodic behavior of diagonal measures and a theorem of Szmerédi on arithmetic progressions, J. d'Analyse Math 31, (1977) 204-256.

[9] F. Takens and E. Verbitski, On the variational principle for the topological entropy of certain non-compact sets, Ergod. Th. and Dynam. Sys. 23, (2003) 317-348..

[10] C. E. Pfister and W.G. Sullivan, On the topological entropy of saturated sets, Ergod. Th. and Dynam. Sys. 27, (2007) 1-29.

[11] D. Thompson, The irregular set for maps with the specification property has full topological pressure, Dynam Sys: An International Journal', 25(1) (2010) 25-51.

[12] P. Walters, An introduction to Ergodic Theory, (Springer-Verlag, Berlin,1982)

[13] X. Zhou and E. Chen, Topological pressure of historic set for $\mathbf{Z}^{d}$-actions, J. Math. Analysis and its applications. 389, (2012) 394-402. 